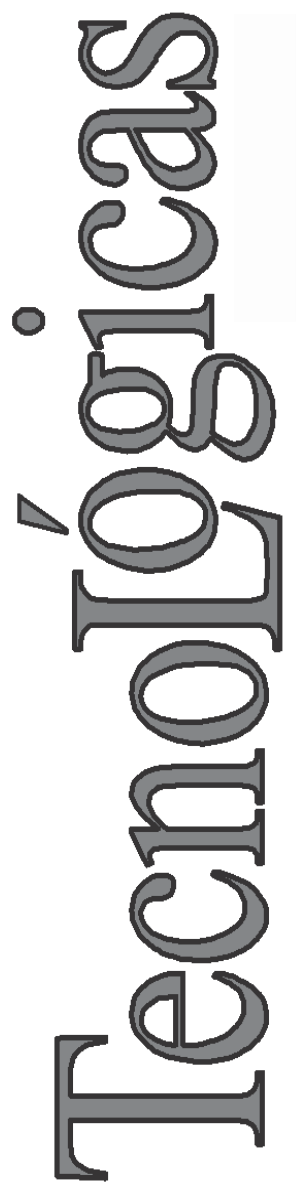

\title{
Resonador de un Cuadrante de Elipse de Microcinta para Filtros en Frecuencias de Microondas
}

\section{A Quarter Ellipse Microstrip Resonator for Filters in Microwave Frequencies}

Samuel A. Jaramillo-Flórez ${ }^{1}$ Sebastián Ramírez-Macías ${ }^{2}$

1 Programa de Ingeniería Electrónica, Escuela de Ingeniería, Universidad del Quindío, Armenia-Colombia samuelangel@uniquindio.edu.co

2 Programa de Ingeniería Electrónica, Escuela de Ingeniería, Universidad del Quindío, Armenia-Colombia 2rami816@gmail.com 


\title{
Resumen
}

Este trabajo describe los resultados obtenidos de la simulación computacional y construcción de resonadores de cuadrante de elipse de microcinta excitados por cintas coplanares para el diseño de filtros en microondas en sistemas de comunicaciones por RF. Utilizando las ecuaciones de la óptica, se explican los fundamentos de la propuesta de esta geometría de resonadores. Se describe la construcción de resonadores de cuadrante de elipse, uno de microcinta y otros dos de cavidad de diferentes tamaños, y un arreglo de cuatro cuadrantes de elipse de microcinta en cascada. Se muestran los resultados de las medidas de los parámetros de dispersión S11 y S21 para identificar las frecuencias de resonancia de los resonadores estudiados, comprobando que estos se comportan en frecuencia como elipses completas por el efecto imagen debido a sus dos espejos en los semiejes mayor y menor, ocupando menos área, y se discuten las posibles aplicaciones.

\section{Palabras clave}

Filtros en microondas; filtros resonadores; parámetros de dispersión; resonador elíptico.

\begin{abstract}
This work describes the results of computational simulations and construction of quadrant elliptical resonators excited by coplanar slot line waveguide for designing microwave filters in $\mathrm{RF}$ communications systems. By means of the equation of optics, are explained the fundamentals of these geometry of resonators proposed. Are described the construction of quadrant elliptical resonators, one of microstrip and other two of cavity, of size different, and an array of four quadrant elliptical resonators in cascade. The results of the measures and the computational calculus of scattering S11 and S21 of elliptical resonators is made for to identify the resonant frequencies of the resonators studied, proving that these have performance in frequency as complete ellipses by the image effect due to their two mirror in both semiaxis, occupying less area, and the possible applications are discussed.
\end{abstract}

\section{Keywords}

Elliptical resonator; microstrip filters; microwaves filters; printed circuits; resonators filters; scattering parameters. 


\section{INTRODUCCIÓN}

Durante las últimas cuatro décadas, los resonadores dieléctri$\cos (D R)$ han llegado a ser un elemento clave en las aplicaciones de filtrado (Wang \& Zaki, 2007). En sistemas de comunicaciones por satélite y móviles se encuentran frecuentemente filtros de guías de onda cargadas con dieléctrico, debido a sus ventajas en términos de su reducción de masa y volumen, bajas pérdidas y estabilidad térmica. En la industria de las comunicaciones móviles terrestres, los costos de los filtros individuales y de la producción en masa son importantes (Mansour, 2004), (Kudsia et al., 1992). Durante las últimas dos décadas, los nuevos sistemas de comunicaciones emergentes han demandado filtros con características más exigentes, habiendo ocasionado un progresos significativo en la tecnología de filtros DR (Mansour, 2009). La rápida expansión de la industria de las comunicaciones inalámbricas ha aumentado la demanda de filtros de microondas para aplicaciones en los equipos móviles y estaciones base.

Se utilizan comúnmente filtros de cavidad coaxial debido a su bajo costo y su desempeño libre de espurios, pero esta clase de filtros poseen valores limitados de factor de calidad (Q), y entonces se debe emplear una tecnología diferente para adaptarse a los nuevo requerimientos de filtrado. Los filtros de resonadores dieléctricos de alta-Q han surgido como dispositivos fundamentales para el diseño de las estaciones-base inalámbricas. El factor de calidad $\mathrm{Q}$ de la cavidad resonadora es dominada por la tangente de pérdidas del material cerámico. Las estructuras como los filtros resonadores dieléctricos cilíndricos planos (Wang et al., 1995) son excitados de diferente manera para mejorar el desempeño con respecto a los espurios de los filtros de modo-dual que operan solamente con modos híbridos.

Utilizando DR de diferentes formas, se pueden realizar filtros de modo triple y cuádruple (Hattori et al., 2003). Este artículo describe el análisis y los resultados de la aplicación del modelo de ondas estacionarias en espejos esféricos en la propagación de microondas en materiales dieléctricos utilizados para construir dispositivos resonadores que tienen topología elíptica, y simulaciones preliminares (utilizando simuladores electromagnéticos 3D) indi- 
can que pueden tener ventajas en la restricción, selección y control de los modos de excitación de los resonadores. Se describen las diferentes maneras de transformar los frentes de onda. Luego se estudia la resonancia en cuadrantes de anillos elípticos excitados por línea de transmisión de par de cintas microstrip coplanares $(C P W)$ y se comprueba que existen comportamientos de resonancia en las estructuras resonantes. Finalmente se presentan las conclusiones y las líneas de trabajo futuro.

\section{METODOLOGíA}

\subsection{Transformación de Frentes de Onda}

Muchas configuraciones no esféricas en circuitos integrados de microondas son de interés práctico pero tienen la desventaja en común de ser difíciles de fabricar. Aun así, se pueden utilizar elementos no esféricos de precisión donde su alto costo se puede justificar. Para construir resonadores en microondas se pueden utilizar las técnicas usadas para concentrar los frentes de onda. Esto permite proponer dispositivos que modifican los frentes de onda para construir filtros. Estos se diseñan a partir de superficies refringentes no esféricas. Se hace que el frente de onda se propague a través de una superficie de separación curva entre dos medios transparentes. Una porción de onda esférica que diverge de una fuente puntual $\mathrm{S}$ se transforma en una onda esférica que converge al punto $\mathrm{P}$, como se muestra en la Fig. 1.

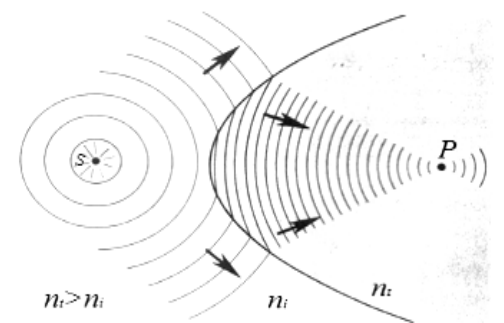

a)

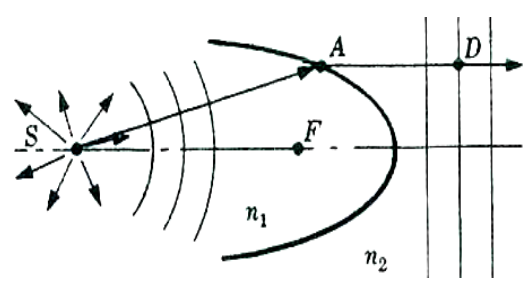

b)

Fig. 1. (a) Superficie ovoide cartesiano refringente. (b) Frentes de onda emergen planos. Fuente: Hecht, E. (1974) 
Los bordes de la onda recorren un trayecto mayor en el medio más denso y alcanzan y pasan la región central de la onda, invirtiendo así el frente de onda. Estas son las superficies ovoide cartesiano (Hecht, 1974). $n_{i}$ y $n_{t}$ son los índices de refracción del medio incidente y transmitido (refringente), respectivamente. Cuando se tiene una fuente puntual sumergida dentro de un medio, como en la Fig. 1(b), donde $n_{1}>n_{2}$, la forma que debe tener la superficie de separación para que los frentes de onda emerjan planos debe ser convexa hacia la derecha, siendo dicha superficie realmente un elipsoide de revolución con excentricidad $e=n_{21}<1$, que corresponde a una superficie de separación elíptica. Si $n_{1}<n_{2}, e>1$ y la curva de la superficie es una hipérbola.

Estas convierten los frentes de onda esféricos originados en una fuente puntual situada sobre el eje en un haz colimado (ondas planas). Si se rebana el extremo elipsoidal de la Fig. 1(b) y se talla en él una superficie hemisférica, y si el punto $S$ es simultáneamente el centro de la esfera como el foco del elipsoide, las ondas no se reflejaran en la primera superficie de separación (hemisférica) y emergerán planas por la superficie elíptica. Un espejo esférico tiene los atributos de las dos configuraciones: parabólica y elíptica. Como parabólica puede formar imágenes de objetos distantes y como elíptica puede formar imágenes de objetos próximos. Los rayos en la Fig. 2 parecen (a) divergir desde o (b) converger hacia los puntos axiales que son los focos geométricos de las superficies curvas.

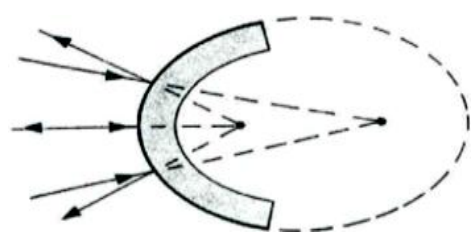

a)

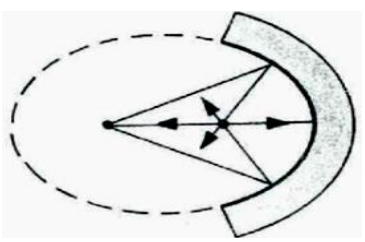

b)

Fig. 2. (a) Diagrama de rayos para un espejo elíptico convexo (a) y elíptico cóncavo (b). Fuente: Hecht, E. (1974)

\subsection{Resonadores Elípticos}

Generalmente, el ancho de banda de un sistema de comunicaciones inalámbrico está comprendido entre 2.4 a $2.5 \mathrm{GHz}$ o de 5.0 a 
$5.8 \mathrm{GHz}$. Para suprimir la interferencia con el sistema de navegación de una aeronave, como los dispositivos portátiles inalámbricos utilizados por los pasajeros, los sistemas inalámbricos usando el mismo espectro electromagnético que la banda asignada a los aviones, las frecuencias debidas a la contaminación electromagnética, etc., deberían disponerse de múltiples filtros rechaza-banda a frecuencias especiales. Se podrían emplear mayor número de celdas resonantes, pero el tamaño del circuito aumentaría inevitablemente. Este inconveniente es resuelto construyendo resonadores de cuadrante de elipse, que ocupa menor área que la elipse completa y conserva sus características en cuanto a las frecuencias de resonancia, puesto que sus semiejes mayor y menor se comportan como espejos y producen imágenes que completan la elipse. La potencia disminuye pero esta se puede aumentar mediante amplificadores.

\section{RESULTADOS Y DISCUSIÓN}

\subsection{Resonador de Cuadrante de Elipse de Cavidad Conductora}

Tamaño pequeño: Se realizó la simulación de un resonador de cavidad de un cuadrante de elipse cuya abertura elíptica tiene un semieje mayor de $5,65 \mathrm{~mm}$ y menor de $2,4426 \mathrm{~mm}$, y está rellena de un compuesto dieléctrico de permitividad eléctrica $\varepsilon_{\mathrm{r}}=500$ y grosor $35 \mathrm{\mu m}$. La estructura se simula sobre sustrato Rogers RO3010 de 1,27 mm de grosor, con $35 \mu \mathrm{m}$ de cobre. Las dimensiones de la guía coplanar han sido halladas con el optimizador de CST STUDIO MICROWAVE, buscando una impedancia de línea de $50 \Omega$ y son 7.2 $\mathrm{mm}$ para el ancho de la pista y $1.41 \mathrm{~mm}$ para el gap, lo que proporciona una impedancia de aproximadamente 49.3 $\Omega$. Las Fig. 3(a) y 3(b) muestran la estructura simulada y la Fig. 3(c) la estructura construida y medida. La Fig. 4(a) muestra la magnitud del parámetro de scattering $S_{11}$ simulado con CST-MICROWAVES SUITES (2010). Se pueden observar las frecuencias de resonancia en 4.2 $\mathrm{GHz}$ y en $8.4 \mathrm{GHz}$, ambas acopladas, lo que facilita el diseño de filtros usando este dispositivo.

En este resonador simulado realmente existen dos resonadores: la cavidad conductora y el resonador dieléctrico, los que determi- 
nan las resonancias encontradas. En la Fig. 4(b) se aprecian los resultados de las medidas del coeficiente de reflexión $\left|S_{11}\right|$ para el resonador cuadrante de elipse construido mostrado en la Fig. 3(c), en donde las frecuencias de resonancia relevantes son 60, 120, $240,360,480,960,1080$ y $1680 \mathrm{MHz}$, que corresponden aproximadamente a múltiplos $(2,3,4,6,8,9$ y 14$)$ de $120 \mathrm{MHz}$, respectivamente. Es de esperarse que a mayores frecuencias presente también resonancias importantes, dado el tamaño reducido del resonador, y basándose en los resultados de la simulación mostrada en la Fig. 4 (a).

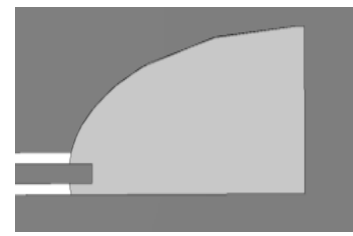

a)

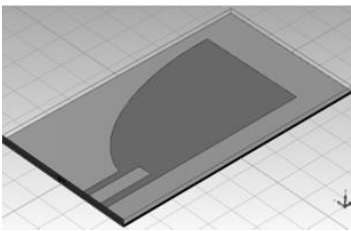

b)

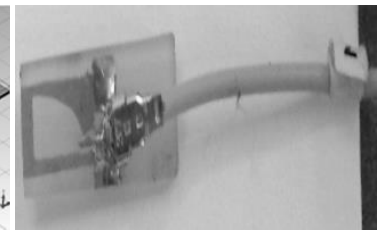

c)

Fig. 3. Configuración del cuadrante de elipse pequeño simulado (a) y (b) y construido (c). Fuente: Autores

Tamaño grande: Se realizó la simulación de un resonador de cavidad de un cuadrante de elipse cuya abertura elíptica tiene un semieje mayor de $13.3235 \mathrm{~cm}$ y menor de $6.5095 \mathrm{~cm}$, ancho del sustrato $=200 \mathrm{~mm}$, longitud del sustrato $=100 \mathrm{~mm}$. La estructura se simula sobre sustrato de baquelita de $1,27 \mathrm{~mm}$ de grosor, con 35 $\mu \mathrm{m}$ de grosor del cobre. Las Fig. 5(a) y 5(b) muestran la estructura simulada y la Fig. 5(c) la estructura construida y medida. La Fig. 6(a) muestra la magnitud del parámetro de scattering $S_{11}$, y se aprecian los resultados de las medidas del coeficiente de reflexión para el resonador cuadrante de elipse de cavidad construido mostrado en la Fig. 5(c), para frecuencias inferiores a 200MHz: 27, 55, $90,112,130,165$ y $190 \mathrm{MHz}$, que corresponden aproximadamente a múltiplos de $27 \mathrm{MHz}$, respectivamente. 


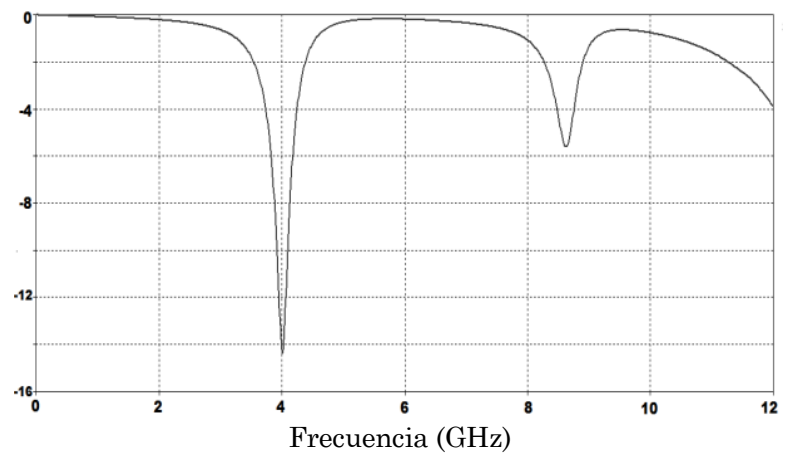

a)

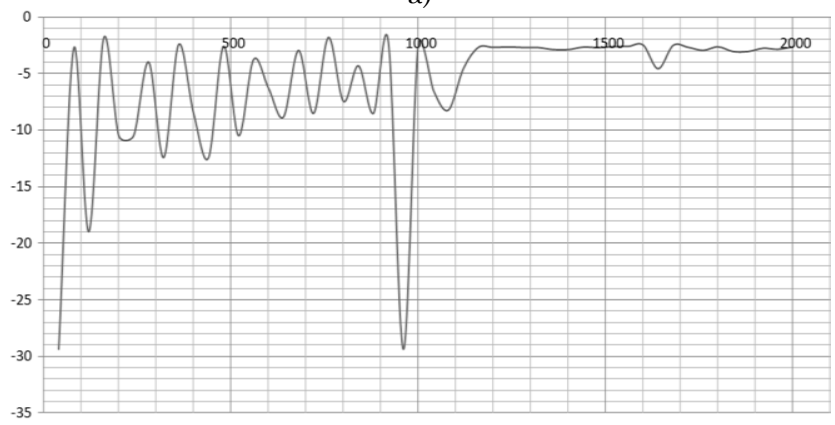

Frecuencia $(\mathrm{MHz})$

b)

Fig. 4. $\left|S_{11}\right|$ simulada (a) y medida (b) en dB, para la estructura de la Fig. 3(a) y 3(c), respectivamente. Fuente: Autores

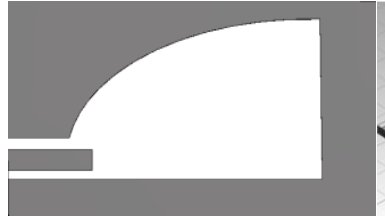

a)

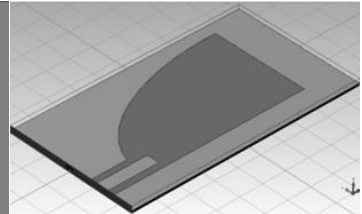

b)

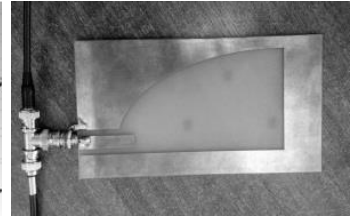

c)

Fig. 5 Configuración del cuadrante de elipse grande simulado (a) y (b), y construido (c). Fuente: Autores

La Fig. 6(c) muestra las frecuencias resonantes medidas para valores mayores a $200 \mathrm{MHz}$ :532, 800,1000, 1200, 1400 y 1888 $\mathrm{MHz}$, que corresponden aproximadamente a múltiplos $(14,21,26$, 32,37 y 50) de $38 \mathrm{MHz}$. Puede verse el parecido con las frecuencias 
de resonancia encontradas con la simulación, que se muestran en la Fig.6(b): 1050, 1300, 1550 y $1850 \mathrm{MHz}$.

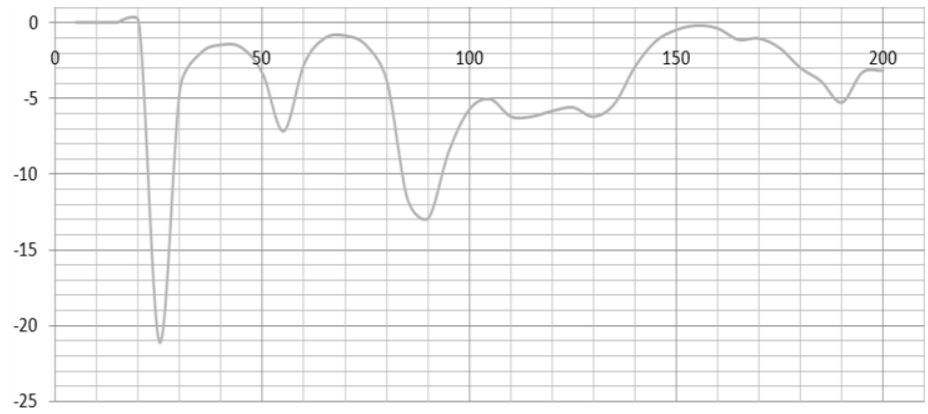

Frecuencia $(\mathrm{MHz})$

a)

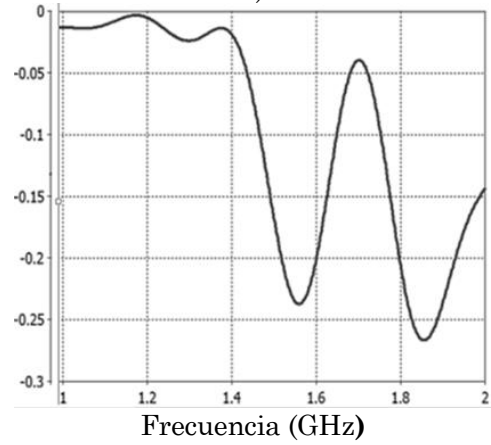

b)

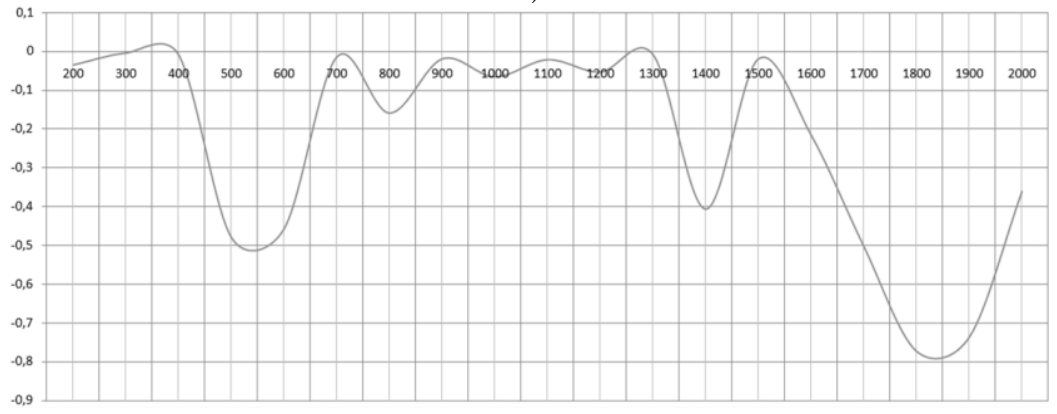

Frecuencia $(\mathrm{MHz})$

(c)

Fig. 6. $\left|S_{11}\right|$ medida (a) $<200 \mathrm{MHz}$ y (c) $>200 \mathrm{MHz}$, y simulada (b), en $\mathrm{dB}$, para la estructura de la Fig. 5(c) y 5(a), respectivamente. Fuente: Autores 


\subsection{Resonador Cuadrante de Elipse en Microcinta}

Se construyó un resonador de cintas de cobre y el sustrato de baquelita que mide $20 \mathrm{~cm}$ de ancho por $10 \mathrm{~cm}$ de largo, como se puede ver en la Fig. 7. Los semiejes de la elipse son $23 \mathrm{~cm}$ eje mayor, y $9 \mathrm{~cm}$ eje menor. La Fig. 8(b) muestra la magnitud de los parámetros de dispersión $S_{11}$ y $S_{21}$ medidos en dB. Se pueden observar las frecuencias de resonancia en 30, 40, (ambas acopladas), 95, 115, (ambas acopladas), 165, 175 y $185 \mathrm{MHz}$, (ambas acopladas) que son aproximadas a las de la Fig. 8(a) obtenidas en la simulación: $44 \mathrm{MHz}$ y $93 \mathrm{MHz}$.

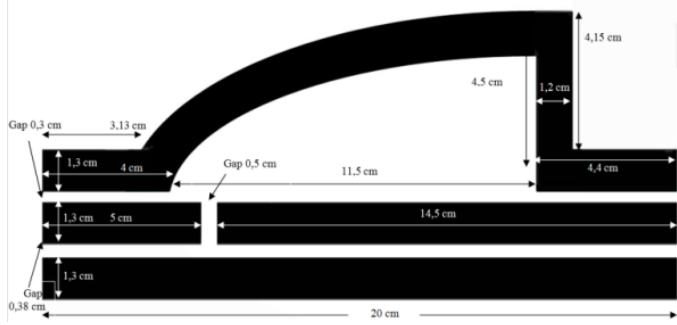

a)

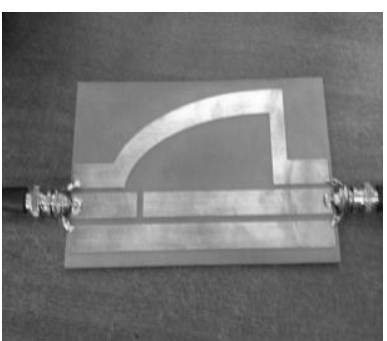

b)

Fig. 7. Dimensiones del cuadrante de elipse (a) y vista superior del dispositivo fabricado (b). Fuente: Autores

\subsection{Cuatro Resonadores de Cuadrante de Elipse en Microcinta}

Se construyó una estructura con cuatro resonadores de cintas de cobre en cascada en un sustrato de baquelita que mide $22 \mathrm{~cm}$ de ancho por $8 \mathrm{~cm}$ de largo, grosor del sustrato $=1.27 \mathrm{~mm}$ y grosor del cobre $=0.035 \mathrm{~mm}$, como se puede ver en la Fig. 9. Los semiejes de la elipse son $30 \mathrm{~mm} \mathrm{~cm}$ eje mayor, y $12.97 \mathrm{~mm}$ eje menor. Las Fig. 10 muestra la magnitud de los parámetros de dispersión $S_{11}$ y $S_{21}$ medidos en $\mathrm{dB}$. Se pueden observar las frecuencias de resonancia en $25,50,90,120,145$ y $155 \mathrm{MHz}$. La simulación predice las frecuencias resonantes superiores a $200 \mathrm{MHz}$ en $725,1220,1640,1880$ y $1990 \mathrm{MHz}$, como se muestra en la Fig. 10(b). 


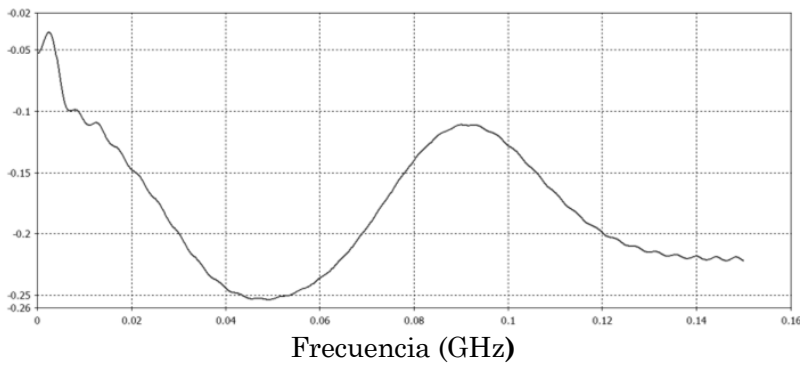

a)

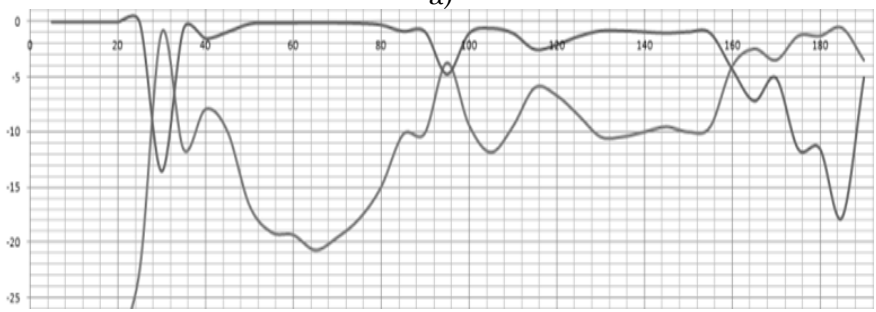

Frecuencia $(\mathrm{MHz})$

b)

Fig. 8. (a) $\left|S_{21}\right|$ simulada (a), en dB, para la estructura de la Fig. 7(a). (b) $\left|S_{11}\right|$ (curva superior) y $\left|S_{21}\right|$ (curva inferior) medida, en $\mathrm{dB}$, para la estructura de la Fig. 7(b). Fuente: Autores

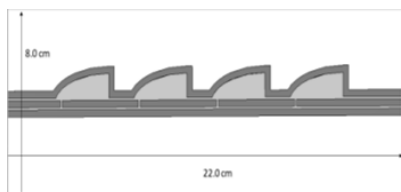

a)

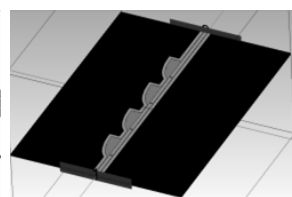

b)

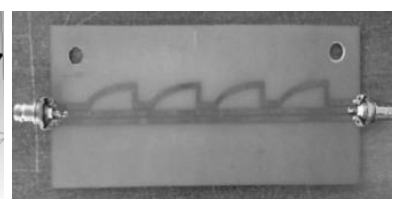

c)

Fig. 9. Configuración de cuatro cuadrantes de elipse simulado (a) y (b), y construido (c). Fuente: Autores

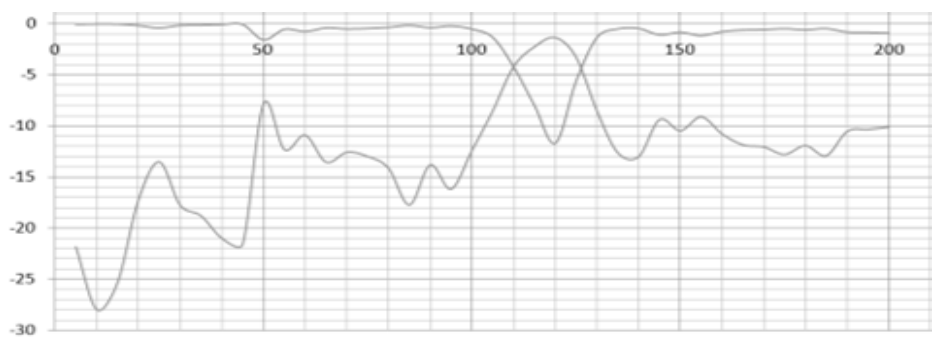

Frecuencia $(\mathrm{MHz})$

a) 


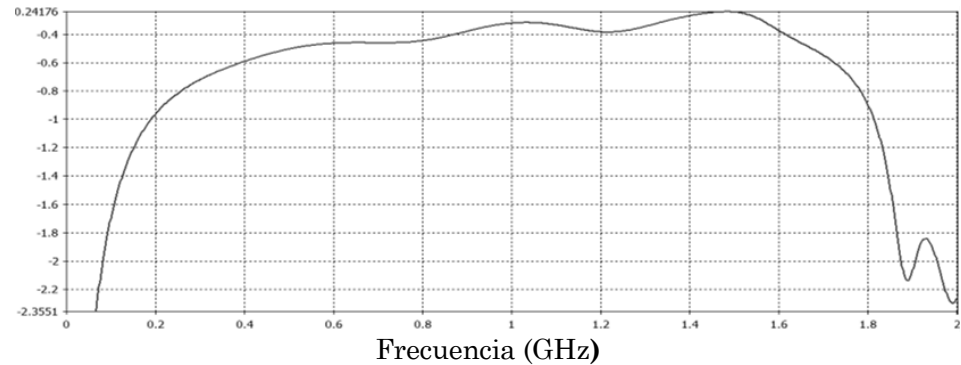

b)

Fig. 10. (a) $\left|S_{11}\right|$ (curva superior) y $\left|S_{21}\right|$ (curva inferior) medida, en $\mathrm{dB}$, para frecuencias $<200 \mathrm{MHz}$ para la estructura de la Fig. 9(c). (b) $\left|S_{11}\right|$ simulada para frecuencias $>200 \mathrm{MHz}$, en dB, para la estructura de la Fig. 9(b). Fuente: Autores

\section{CONCLUSIONES}

Se han propuesto resonadores basados en cuadrantes de elipse en microcinta, como alternativa para reducir el tamaño de los resonadores sin perder las ventajas de la gran gama de frecuencias resonantes que posee el resonador elíptico comparado con el circular, reduciendo a la cuarta parte el área ocupada en el circuito impreso. Se demostró que se generan múltiples frecuencias resonantes en las estructuras presentadas, y estas pueden ser utilizadas para diseñar y construir filtros de banda estrecha, banda ancha y multibanda, específicamente en las aplicaciones de VHF y la parte baja de UHF, como es el caso de las comunicaciones móviles. Para ello se requiere el empleo de la metodología de las resonancias acopladas con cuadrantes de elipse en cascada de diferentes tamaños y un programa computacional (en construcción) que determina la frecuencia de resonancia de la estructura elíptica dadas sus dimensiones y características de los materiales.

La idea original aquí presentada de utilizar resonadores de cuadrante de elipse con el campo eléctrico solo tangencial a la superficie pueden usarse para construir arreglos que se comportan como metamateriales. Para mejorar la respuesta de estos resonadores, se puede cubrir la región en el interior de la sección de elipse de una fina capa dieléctrica de alto valor de permitividad eléctrica, por ejemplo $\mathrm{TiO}_{2}$ o $\mathrm{BaTiO}_{2}$. Se logra un descenso apreciable de todas las frecuencias resonantes, puesto que se introducen resona- 
dores dieléctricos que interactúan con los de microcinta y cavidad conductora, y se podrán diseñar filtros pasa-bajo, pasa-alto, pasabanda y rechaza-banda livianos y de tamaño reducido, en VHF.

\section{AGRADECIMIENTOS}

Los autores agradecen a J. Atehortua-Ramos, a L. M. Castañeda-Herrera.

\section{REFERENCIAS}

Hattori, J., Wada, T., Kubo, H. and Ishikawa, Y. (2003), 2GHz band Quadruple Mode Dielectric Resonator Filter for Cellular Base Stations, IEEE MTT-S Int. Microwave Symp Dig., (Philadelphia, PA), 933-936.

Hecht, E. (1974). Óptica, McGraw-Hill, Mexico.

Kudsia, C., Cameron, R. and Tang, W.C. (1992). Innovations in Microwave Filters and Multiplexing Networks for Communications Satellite Systems, IEEE Trans. Microwave Theory Tech., vol. 40, pp. 11331149 .

Mansour R. R., (2004). Filter Technologies for Wireless Base Stations, IEEE Microwave Magazine, 68-74.

Mansour, R. R. (2009). High-Q Tunable Dielectric Resonator Filters, IEEE Microwave Magazine, 84-98.

Wang C. and Zaki K. A. (2007). Dielectric Resonators and Filters, IEEE Microwave Magazine, 115-127.

Wang, C., Tao, H.W., Zaki, K. A. and Mansour, R. R. (1995). Mixed Modes Cylindrical Planar Dielectric Resonator Filters with Rectangular Enclosure, IEEE Trans. Microwave Theory Tech., vol. 43, 817-2823. 